\title{
Hegel est-il «pragmatiste»? Quelques remarques critiques à propos d'un problème mal posé
}

\author{
OLIVIER TINLAND Université Paul-Valéry Montpellier 3
}

RÉSUMÉ : La question «Hegel est-il pragmatiste?» a fait l'objet de nombreux débats chez les spécialistes de la philosophie hégélienne et du pragmatisme. Dans cet article, je me propose de clarifier les termes du problème et d'évaluer les forces et les faiblesses de deux manières de répondre à cette question : la première consiste à repérer des thèmes pragmatistes dans la pensée hégélienne, la seconde consiste à identifier un critère minimal et négatif de rapprochement entre l'hégélianisme et le pragmatisme. À la lumière de cet examen critique, il apparaît que toute approche générale de cette question semble vouée à l'échec.

ABSTRACT: The question "Is Hegel a pragmatist?" has been the subject of much debate among specialists in Hegelian philosophy and pragmatism. In this article, I intend to clarify the terms of the problem, and to assess the strengths and weaknesses of two ways of answering this question: the first is to make explicit pragmatic themes in Hegelian thought, and the second is to identify a minimal and negative criterion of rapprochement between Hegelianism and pragmatism. In light of this critical examination, it appears that any general approach to this question is doomed to failure.

Mots clés : Hegel, pragmatisme, Rorty, Peirce, Dewey, Brandom

Dialogue 59 (2020), 271-303

(C) The Author(s), 2020. Published by Cambridge University Press on behalf of the Canadian Philosophical Association/l'Association canadienne de philosophie.

doi:10.1017/S0012217320000219 
"Once one starts to look for pragmatism

in Hegel, one finds quite a lot to go on.»

Richard Rorty, «Dewey between Hegel and Darwin» (1998)

\section{Le problème de départ : que veut dire «Hegel pragmatiste»?}

La publication des œuvres majeures de Richard Rorty et de Robert Brandom dans les années 1980-1990 fut l'occasion de la naissance d'un champ de recherche qui n'a depuis cessé de gagner en ampleur et en complexité : l'interprétation (et la réactualisation) de la pensée hégélienne à l'aune de l'histoire de la philosophie anglo-américaine du $\mathrm{XX}^{\mathrm{e}}$ siècle (plus précisément de la fin du $\mathrm{XIX}^{\mathrm{e}}$ siècle jusqu'au début du $\mathrm{XXI}^{\mathrm{e}}$ siècle $^{1}$ ), en particulier à l'aune de la constitution, du développement et des transformations du mouvement pragmatiste, depuis les premières formulations de Charles Sanders Peirce, William James, John Dewey et George Herbert Mead jusqu'aux développements les plus récents qu'en ont proposés Richard Bernstein, Hilary Putnam, Richard Rorty, Joseph Margolis et Robert Brandom².

La question d'un «pragmatisme» de Hegel — désormais considérée, de façon assez surprenante, comme un lieu commun des débats contemporains (notamment dans le monde anglophone, mais aussi en Allemagne ou en Italie par exemple) — s'avère, de fait, particulièrement sujette à controverse, dans la mesure où elle enveloppe plusieurs questions elles-mêmes hautement disputées, qui n'ont pas toujours (loin s'en faut) fait l'objet d'un traitement suffisamment explicite, nuancé et approfondi.

Parmi ces questions préalables, citons (au risque de paraître trivial) celles qui s'imposent immédiatement à un esprit non prévenu : comment définir le pragmatisme? Est-il possible de dépasser l'éclatement manifeste des tendances apparentées à cette étiquette dont l'unité nominale peut s'avérer trompeuse ${ }^{3}$ et quelles seraient les caractéristiques principales d'un éventuel noyau commun à ces tendances? Est-il possible de faire se recouper les engagements philosophiques des grandes figures du pragmatisme classique et contemporain sur des thèses ou des démarches admises ou promues par l'ensemble (ou à tout le moins une grande majorité) de ces figures? Mais aussi, si l'on se tourne vers l'autre terme de la relation : quelles sont les caractéristiques fondamentales de

1 Voir par exemple : Angelica Nuzzo, Hegel and the Analytic Tradition (2010); Paul Redding, Hegel and the Return of Hegelian Thought (2007); Tom Rockmore, Hegel, Idealism, and Analytic Philosophy (2005).

2 Sur l'essor récent du thème «Hegel et le pragmatisme», on pourra se reporter au dossier réuni dans le numéro «Hegel pragmatiste?» (dir. J.-M. Buée, E. Renault, O. Tinland et D. Wittmann) de la revue Philosophie, $\mathrm{n}^{\circ} 99$ (voir Buée et al., 2008). On rappellera, pour mémoire, l'intitulé célèbre de l'article d'Arthur Lovejoy, «The Thirteen Pragmatisms» (Lovejoy, 1908). 
la pensée hégélienne? Que peut-on considérer comme des engagements philosophiques typiquement et spécifiquement «hégéliens»? Est-il possible d'opérer une reprise sélective de certains de ces engagements (au détriment d'autres engagements jugés secondaires, anachroniques ou indésirables) sans porter atteinte à la cohérence profonde (ou supposée telle) de l'hégélianisme ${ }^{4}$ ? Et encore : pourquoi faire porter l'interrogation spécifiquement sur Hegel? En quoi la question d'un «pragmatisme» de Hegel (ou, inversement, d'un «hégélianisme» des pragmatistes) s'impose-t-elle avec une pertinence particulière? Pourquoi pas un «Kant pragmatiste», ou un Fichte, un Nietzsche, un Heidegger, voire un Leibniz ou un Spinoza ${ }^{5}$ ?

Faute de clarifier de tels présupposés, il semble difficile de proposer une réponse claire et indiscutable à la question du «pragmatisme» de Hegel, qui échappe à l'imprécision, à la partialité et à l'arbitraire des prémisses herméneutiques assumées par tel ou tel commentateur, et plus largement aux effets de mode intellectuelle qui conduisent régulièrement à repeindre les vieux modèles de la tradition philosophique aux couleurs clinquantes dictées par le goût du jour. Hegel lui-même a pu être successivement interprété (liste non exhaustive) comme un anticipateur (contrarié) de l'existentialisme ${ }^{6}$, comme un précurseur (contrarié) du marxisme ${ }^{7}$, comme le promoteur d'une «raison élargie» dans laquelle devrait s'ancrer la phénoménologie

4 À ce sujet, voir la mise au point décisive de Rolf-Peter Horstmann, «What is Hegel's Legacy and What Should We Do With It?» (1999, p. 275-287). L'auteur répond par la négative à cette question, arguant du caractère «monolithique» du système hégélien qui contraint à «l'avaler en totalité ou pas du tout».

5 Une telle question n'est pas purement rhétorique, puisque Robert Brandom a tenté, dans Tales of the Mighty Dead (2002, voir ch. 4 et 5), d'inscrire sa propre version du pragmatisme dans une généalogie philosophique incluant notamment Spinoza et Leibniz, mais aussi Frege et Heidegger. Pour une interprétation pragmatiste de Heidegger, voir Mark Okrent, Heidegger's Pragmatism. Understanding, Being, and the Critique of Metaphysics (1988), ainsi que Richard Rorty, Essais sur Heidegger et autres écrits (1995, $1^{\text {ère }}$ partie). À la suite de l'ouvrage pionnier de René Berthelot, Un romantisme utilitaire. Étude sur le mouvement pragmatiste (1911), les affinités entre Nietzsche et le pragmatisme (notamment celui de James) ont été amplement thématisées par Jean Granier dans Le problème de la vérité dans la philosophie de Nietzsche (1966) puis, plus récemment, et de manière plus rigoureuse, par Pietro Gori dans Il pragmatismo di Nietzsche. Saggi sul pensiero prospettivistico (2016). Bizarrement, Fichte, penseur par excellence du primat du pratique sur le théorique, n'a pas fait l'objet d'une lecture «pragmatiste», si l'on excepte l'article déjà ancien d'Ellen Bliss Talbot, «The Philosophy of Fichte in its Relation to Pragmatism» (1907).

6 Voir Jean Wahl, Le malheur de la conscience dans la philosophie de Hegel (1929).

7 Voir Alexandre Kojève, Introduction à la lecture de Hegel (1947). 
contemporaine pour accomplir toutes ses promesses ${ }^{8}$, comme un glorieux ancêtre de la critique communautarienne du libéralisme ${ }^{9}$ ou comme l'instaurateur précoce d'une philosophie politique normative de la reconnaissance intersubjective $^{10}$. La question est de savoir ce que pourrait apporter, par rapport à l'ensemble de ces interprétations, une lecture de Hegel en grand ancêtre du pragmatisme (ou du pragmatisme en rejeton tardif de l'hégélianisme). Pour y voir plus clair, on envisagera dans les pages qui suivent deux stratégies différentes visant à opérer sinon une identification, du moins un rapprochement entre les deux pôles du rapport «Hegel/Pragmatisme».

\section{Une première stratégie : le repérage de thèmes «pragmatistes» dans l'œuvre de Hegel}

La difficulté d'établir une interprétation et une évaluation de la pensée de Hegel qui soit commune aux principaux représentants du pragmatisme peut conduire à envisager une première stratégie, qui consiste à repérer des caractéristiques (supposément) centrales de la pensée hégélienne pour en faire émerger des thèmes (prétendument) «pragmatistes». Nous prendrons pour exemple d'une telle stratégie un article d'Antje Gimmler intitulé «Pragmatic Aspects of Hegel's Thought»" ${ }^{11}$, article qui a le mérite d'illustrer (volontairement mais aussi involontairement) un certain nombre de problèmes liés à une telle stratégie.

Constatant le net retour en grâce du maître de Berlin chez les tenants du «néopragmatisme ${ }^{12} »$, Gimmler s'interroge avec raison sur les présupposés d'un tel phénomène : «Qu'est-ce qui est pragmatique chez Hegel? Hegel

8 Voir Maurice Merleau-Ponty, «L'existentialisme chez Hegel», dans Sens et nonsens (1948).

9 Voir Charles Taylor, Hegel et la société moderne (1998).

10 Voir Axel Honneth, Les pathologies de la liberté. Une réactualisation de la philosophie du droit de Hegel (2008).

11 Inclus dans le volume dirigé par William Egginton et Mike Sandbothe, The Pragmatist Turn in Philosophy (Gimmler, 2004). Sauf indication contraire, les traductions sont de moi.

12 La liste proposée par Gimmler est suffisamment vaste pour poser, en elle-même, de redoutables problèmes d'homogénéité doctrinale : Rescher, Rorty, Putnam, Habermas, Brandom, Davidson, à quoi il faut ajouter, selon l'auteure, Wittgenstein, Sellars et Quine. La définition qu'elle donne du néopragmatisme est elle aussi suffisamment large et accueillante pour s'accommoder d'une telle disparité : «Le terme "néopragmatisme" renvoie au mouvement philosophique contemporain, à multiples facettes, qui reconsidère et réutilise des thèmes et des éléments de base du pragmatisme classique américain dans le contexte du tournant linguistique, c'est-à-dire au service d'une recherche sur le langage et la signification conçus comme dépendants de l'usage et de la pratique» (2004, p. 47). On pourrait s'interroger sur la valeur de démarcation d'une telle définition. 
est-il un penseur authentiquement pragmatiste?» (2004, p. 48) Pour répondre à ces questions, l'auteur, concédant qu' «il n'existe aucune détermination précise, communément partagée, de ce qui peut être compris comme "pragmatisme"», procède d'abord (1) à une «typologie du pragmatisme afin de clarifier la signification des termes "pragmatique" et "pragmatisme" tels qu'ils sont utilisés dans les débats actuels», puis (2) «discute deux interprétations néopragmatistes de Hegel» (celles de Richard Rorty et Robert Brandom), avant de (3) faire porter l'accent sur des «éléments de l'antireprésentationalisme pragmatique de Hegel eu égard à sa critique de l'épistémologie», en particulier sur l'usage d'un «concept d'expérience» dégagé des dogmes de l'empirisme, propre à favoriser une interprétation pragmatiste de l'auteur de la Phénoménologie de l'esprit (ibid., p. 48) qui permette, par contrecoup, de se libérer des «restrictions néopragmatistes» en mettant l'accent non seulement sur la dimension langagière de l'action, mais aussi sur sa dimension «poïétique» (ibid., p. 49). Passons en revue ces trois moments de l'argumentation avant d'en dresser un bilan critique.

(1) Quelles caractéristiques du pragmatisme retenir si l'on veut s'enquérir de l'existence de thèmes «pragmatistes» dans la pensée de Hegel? Gimmler insiste en premier lieu (en concédant volontiers le caractère trop vague d'un tel critère) sur le primat explicatif de la pratique sur la théorie, du «knowing how» sur le «knowing that» ${ }^{13}$, primat qui implique selon elle diverses formes $\mathrm{d}^{\prime}$ antireprésentationalisme ${ }^{14}$ (c'est-à-dire de rejet de la thèse de l'existence d'une réalité indépendante de l'esprit humain que celui-ci devrait s'efforcer de représenter adéquatement par ses idées ou «représentations»), notamment l'opposition au «représentationalisme plutôt mental et épistémologique» (Gimmler, 2004, p. 49) du rationalisme (Descartes) et de l'empirisme (Locke) modernes, ainsi que l'opposition au représentationalisme inhérent au «constructivisme kantien», sans oublier leurs corrélats parasitaires, le scepticisme moderne et le relativisme (ibid., p. 50) ${ }^{15}$. Face au «théoricisme» dominant de la tradition représentationaliste, le pragmatisme, à l'instar de Dewey, remettrait en question le «primat de la théorie sur la pratique», saperait les bases de la

13 On sait que cette distinction ne provient pas du pragmatisme, mais de Gilbert Ryle, The Concept of Mind (1949, $2^{\mathrm{e}}$ partie, p. 27 sqq). Elle a fait l'objet d'une intégration à l'univers pragmatiste dans Rendre explicite de Robert Brandom.

14 Notons d'emblée le caractère problématique du recours — forcément anachronique — à une notion qui n'apparaît sur la scène pragmatiste que tardivement, dans l'œuvre de Richard Rorty (voir l'introduction à Objectivisme, relativisme et vérité [Rorty, 1994, p. 7-25]). Si le terme apparaît aussi dans d'autres contextes (notamment la théorie de l'énaction), il est très probable que l'auteure s'inspire ici de l'usage rortyen.

15 Une telle manière de présenter les choses est manifestement tributaire de la lecture de la philosophie moderne présentée par Rorty dans La philosophie et le miroir de la nature (2017, ch. 1 et 3 ). 
«spectator's theory of knowledge» pour échafauder une «théorie de l'expérience et de la connaissance qui englobe la cognition et l'action» (ibid., p. 51). Le concept de «pratique» ici à l'œuvre peut être entendu soit, comme chez Dewey, en un sens «instrumental» (dans un tel cas, le langage sera un outil pratique parmi d'autres, fût-il «the tool of tools»), soit, comme chez Brandom ou Habermas, en un sens «normatif» (dans cet autre cas, la pratique instrumentale dérive au contraire de la pratique langagière intersubjective et des normes qu'elle mobilise et rend explicites) ${ }^{16}$. Quant à la «théorie», elle peut consister soit dans une «reconstruction de la pratique» (Brandom) jouant un rôle «fondamental» par rapport à celle-ci, soit dans une activité conditionnée et fonctionnelle soumise aux mêmes réquisits pratiques que les autres activités, tout en étant capable d'opérer un retour réflexif sur ses propres conditions et fonctionnalités (Dewey).

(2) Nous ne présenterons pas ici en détail la discussion, par Gimmler, des interprétations de Rorty et Brandom. Contentons-nous de souligner les critiques qu'elle leur adresse. D'un côté, Rorty, qui insiste de manière unilatérale (en jetant par-dessus bord la théorie de l'esprit absolu) sur la «détranscendantalisation de la raison» opérée par Hegel moyennant un double geste (typiquement kuhnien) d'historicisation et de contextualisation de celle-ci (Gimmler, 2004, p. 54) ${ }^{17}$, néglige volontairement la théorie de la connaissance de Hegel, en particulier sa théorie de l'expérience, en raison de sa défiance à l'égard des relents métaphysiques du concept deweyen d'expérience ${ }^{18}$. De l'autre, Brandom, qui se montre davantage soucieux de reconstruire un lien étroit entre l'idéalisme hégélien et sa propre version (rationaliste, linguistique et normative) du pragmatisme, commet l'erreur de réduire le concept hégélien de pratique à celui de pratique linguistique de reconnaissance intersubjective : ainsi se trouve passée sous silence l'existence d'un «concept plus large de pratique, qui inclut l'intersubjectivité et l'action poïétique», la reconnaissance et «l'appropriation, c'est-à-dire l'extériorisation, l'objectivation ou la transformation» (ibid.,

16 Sur la confrontation de ces deux démarches (dans des perspectives opposées), voir David Hildebrand, Beyond Realism and Antirealism: John Dewey and the Neopragmatists (2003) et Robert Brandom, Perspectives on Pragmatism (2011).

17 Voir Rorty, Conséquences du pragmatisme (1993a, ch. 5, p. 188) : «Par "hégélien”, je veux dire ici considérer les développements culturels que la philosophie, pour Kant, avait le devoir de conserver et de protéger, comme de simples étapes transitoires de l'Esprit-monde». Rorty résume ce qui constitue selon lui l'apport hégélien au pragmatisme dans la formule «la philosophie est son temps saisi dans la pensée» (ibid., «Introduction», p. 58) et soutient que cette «temporalisation de la raison» est «l'unique pas décisif dont la méfiance pragmatiste envers la philosophie est issue» (ibid., p. 60).

18 Pour une présentation de la critique rortyenne du concept deweyen d'expérience mobilisé dans Expérience et Nature, voir Conséquences du pragmatisme (Rorty, 1993a), ch. 5 : «La métaphysique de Dewey». 
p. 56), non seulement la relation interpersonnelle, mais aussi la relation du sujet à un monde objectif. Une telle sous-estimation du concept hégélien d'expérience, selon Gimmler, outre qu'elle amputerait la pensée hégélienne de l'une de ses dimensions essentielles, nous ferait manquer le trait le plus typiquement «pragmatiste» de Hegel.

(3) Dans un dernier moment, il est donc nécessaire de proposer une présentation plus complète d'un tel concept d'expérience afin de mettre en évidence le «pragmatisme» profond qui sous-tend son élaboration. En s'appuyant sur l'introduction à la Phénoménologie de l'esprit — qui constitue un morceau de choix pour les interprètes pragmatistes de Hegel — Gimmler décèle une critique de la théorie moderne de la connaissance entendue comme la tentative d'évaluer les conditions de possibilité de la connaissance antérieurement à toute connaissance effective, notamment de l'assimilation de la connaissance à un «instrument intervenant entre "nous" et "l'objet"» et de la croyance en la «possibilité d'examiner l'outil indépendamment de son usage» (2004, p. 58). $\mathrm{Au}$ contraire, pour Hegel, «la connaissance devient une partie d'un concept global d'expérience» ou «d'un processus d'expérience dans lequel objet et concept représentent les produits changeants et extériorisés d'une interaction» (ibid.). Plutôt que de se tenir en amont de l'expérience, «l'opération cognitive s'intègre à l'objet; la connaissance d'un objet est obtenue par un processus; il s'agit d'une connaissance en constante autocorrection et expansion». En outre, «si la pensée est conceptualisée comme interagissant avec son environnement, si ses concepts ne sont formés que par cette interaction, et si son activité produit une appropriation et une transformation, alors son objectif n'est pas contemplatif mais pratique» (ibid., p. 59).

Une telle conception générale de l'expérience, qui n'est pas sans affinités avec celles de James et (surtout) de Dewey, trouve, selon l'auteur, à s'illustrer de manière emblématique dans la section de la Phénoménologie de l'esprit consacrée à la dialectique de la maîtrise et de la servitude, dont Brandom aurait négligé le versant qui concerne la critique de la théorie classique de la connaissance (au profit du seul thème de la reconnaissance intersubjective ${ }^{19}$ ). Au-delà du thème de la lutte pour la reconnaissance, l'intérêt de cette section consisterait à articuler une «pratique de l'intersubjectivité» à une «pratique poïétique» (celle du travail de la chose par le serviteur) pour rendre compte de la «certitude de soi» de la subjectivité : le moment d'«extériorisation et d'objectivation concrètes», incarné par le travail du serviteur, constitue une alternative à la «relation contemplative à la chose» qu'assume le maître (Gimmler, 2004, p. 61). Ainsi s'amorcerait «une théorie de la conscience de soi authentiquement

19 Cette lecture intersubjectiviste est présentée dans Robert Brandom, «La structure du désir et de la reconnaissance : conscience de soi et autoconstitution», dans D. Perinetti et M.-A. Ricard, dir., La Phénoménologie de l'esprit de Hegel : lectures contemporaines (2009). 
pragmatique» rompant avec le modèle contemplatif du représentationalisme moderne : l'articulation de la reconnaissance intersubjective et de la relation objectivante de transformation de la chose extérieure permettrait de conquérir un concept non mentaliste de conscience de soi, défini selon le double axe de ces deux types d'activités complémentaires que sont le langage et le travail ${ }^{20}$. Pour peu que l'on minore l'importance de l'esprit absolu (qui constitue de ce point de vue une retombée inconséquente dans le modèle contemplatif de la connaissance $)^{21}$, il devient loisible de soutenir qu' «une théorie pragmatique de l'expérience, de la connexion entre connaissance et action, peut être élaborée à partir de la reconstruction - rendue possible par le tournant antireprésentationaliste de Hegel — de nos prétentions à la connaissance dans des contextes concrets de pratique humaine» (ibid., p. 62). Le «pragmatisme» de Hegel devient tangible, une fois que l'on a placé sa critique de la théorie classique de la connaissance au cœur de sa propre démarche philosophique.

L'interprétation du concept hégélien d'expérience qui est ici proposée constitue assurément un moyen fécond de favoriser un rapprochement entre l'auteur de la Phénoménologie de l'esprit et certaines figures majeures du pragmatisme (principalement celles qui s'inscrivent dans le sillage instrumentaliste de Dewey). On peut néanmoins soulever plusieurs problèmes qui rendent périlleuse une telle tentative de penser le «pragmatisme» de Hegel à partir de la conception de l'expérience présentée dans son premier grand ouvrage : (1) la focalisation sur la seule Phénoménologie de l'esprit au détriment du reste de l'œuvre de Hegel, (2) la focalisation, au sein de cette œuvre, sur la seule dialectique du maître et du serviteur au détriment des autres «configurations de la conscience», (3) l'attribution à Hegel d'une position antireprésentationaliste, (4) la restriction du «pragmatisme» à certains de ses représentants (James, Dewey) au détriment des autres. Évoquons-les brièvement afin de mettre en évidence les failles d'une telle stratégie interprétative.

(1) En choisissant d'interpréter la pensée hégélienne au seul prisme de la Phénoménologie de l'esprit, Antje Gimmler, à l'instar d'autres pragmatistes

20 L'influence de l'interprétation de la Realphilosophie d'Iéna proposée par Jürgen Habermas est ici manifeste : voir «Travail et interaction. Remarques sur la Philosophie de l'esprit de Hegel à Iéna», dans La technique et la science comme "idéologie" (1973) et «Manières de détranscendantaliser. De Kant à Hegel et retour», dans Vérité et justification (2001).

21 Voir Gimmler (2004, p. 62) : «Tandis que Hegel procède de manière antireprésentationaliste dans sa critique de la théorie de la connaissance, il ne tire pas la même conséquence pour la compréhension et la définition du statut de sa propre philosophie». Là encore, il s'agit d'une réminiscence assez nette des critiques de Habermas. 
contemporains $^{22}$, mais aussi d'un vaste pan de la réception francophone de Hegel $^{23}$, propose une reconstruction extrêmement partielle et biaisée de celle-ci, en particulier de la conception de l'expérience et de la connaissance qui s'y trouve exposée. La conception de l'expérience que Hegel propose dans la Phénoménologie de l'esprit est encore très spécifique et ne saurait valoir pour sa conception achevée : elle a essentiellement un rôle négatif (justifier le renoncement de la conscience à ses repères épistémiques ordinaires et son élévation progressive au-dessus de son propre point de vue, jusqu'à celui du «savoir absolu») et n'opère qu'au niveau («phénoménologique») de la conscience, non au niveau («psychologique») de l'esprit en tant que tel. Il s'agit en outre ici d'une expérience fort peu «empirique», délestée de sa contingence et de sa particularité, stylisée et logicisée de manière à épouser les contours (nécessaires et universels) du mouvement dialectique de la conscience vers le savoir absolu : on est donc loin de la contextualisation empirique de la connaissance philosophique qu'un Dewey, par exemple, a pu proposer ${ }^{24}$.

En outre, la critique de la théorie de la connaissance (qui est suggérée en quelques pages dans l'introduction de l'ouvrage, plutôt qu'élaborée en détail) correspond elle aussi à un objectif spécifique de méthode, non à une thèse de portée générale : il s'agit de justifier la stratégie méthodologique de la Phénoménologie de l'esprit (présenter l'émergence des normes de la connaissance à même l'expérience dialectique de la conscience) en référence à certaines thèses spécifiques (principalement celles de Kant et de Reinhold), certainement pas de statuer sur l'ensemble de la théorie de la connaissance telle qu'elle a pu être présentée dans la tradition philosophique. La critique de la connaissance

22 Citons les cas exemplaires de Rorty (Contingence, ironie et solidarité [1993b]) et de Brandom (A Spirit of Trust. A Reading of Hegel's Phenomenology [2019]).

23 Un tel privilège accordé à la Phénoménologie de l'esprit dans la philosophie française au $\mathrm{XX}^{\mathrm{e}}$ siècle est lié pour partie à des raisons idéologiques (pensons à l'interprétation «marxiste» de Kojève, dont l'influence sera profonde et durable), pour partie à des raisons éditoriales (la Phénoménologie de l'esprit fut pendant longtemps le seul ouvrage majeur de Hegel ayant fait l'objet d'une traduction fiable et d'un commentaire approfondi, par Jean Hyppolite). On retrouvera cette focalisation jusque chez les plus anti-hégéliens des disciples et lecteurs de Kojève et d'Hyppolite, notamment chez Sartre (L'Être et le néant), Deleuze (Nietzsche et la philosophie) et Foucault (qui consacre en 1949 son mémoire de maîtrise à la "Constitution d'un transcendantal historique dans la Phénoménologie de l'esprit de Hegel»).

24 À ce sujet, voir Gérard Deledalle, L'idée d'expérience dans la philosophie de Dewey (1967). Cela n'exclut pas que l'expérience fasse parfois l'objet d'une idéalisation philosophique chez Dewey lui-même, notamment dans Expérience et nature : voir en ce sens Richard Rorty, «La métaphysique de Dewey», dans Conséquences $d u$ pragmatisme (1993a). 
comme simple «instrument» ou «médium» ${ }^{25}$ censé permettre à un sujet de combler le fossé qui le sépare de l'objet n'est qu'un aspect, très limité, de la manière dont Hegel conçoit la connaissance (ordinaire, scientifique, philosophique) dans le système de la maturité et dont il critique corrélativement les conceptions kantienne et postkantienne de la connaissance.

(2) Ce biais de lecture s'avère d'autant plus problématique que l'auteure l'aiguise encore en accordant une place privilégiée à une section isolée de l'ouvrage, la fameuse «dialectique du maître et du serviteur». Ainsi que je l'ai précisé ailleurs ${ }^{26}$, une telle stratégie de lecture comporte de multiples écueils. Elle implique d'une part de séparer arbitrairement une partie du Tout, contredisant l'intention explicitement systématique de Hegel; elle revient en outre à absolutiser la portée relative de ce qui n'est qu'une section subalterne de l'œuvre, en négligeant les enjeux plus généraux de cette section, une fois celle-ci replacée dans son contexte; elle conduit enfin à accorder une signification positive (la libération du serviteur par le travail) à une dialectique qui a une issue essentiellement négative et marque une forme d'échec (provisoire) de la conscience de soi à obtenir un savoir adéquat d'elle-même ${ }^{27}$. Last but not least, la mise en exergue du rôle du travail dans le rapport du serviteur au maître laisse entendre qu'il s'agirait ici d'une valorisation de la pratique (ou de la dimension «poïétique» de l'expérience) par rapport à la théorie (ou à l'«activité contemplative»), alors même que toutes les expériences de la conscience ne sont appréhendées dans cet ouvrage que sous l'angle - certes non exclusif — d'un accès au savoir. Le travail du serviteur (envisagé dans le cadre d'une «parabole» conceptuelle détachée de tout contexte socio-historique) n'intéresse ici Hegel que parce qu'il est un vecteur non pas de liberté, mais de vérité : il s'agit pour le serviteur d'accéder à la certitude de ce qu'il est, à la conscience de la structure véritable de la conscience de soi, non à une quelconque émancipation à l'égard de la nature ou de la domination sociale (quoi qu'ait pu en dire Kojève). Nous sommes donc ici très éloignés de la thèse «pragmatiste» d'un bouleversement de la hiérarchie de la théorie et de la pratique : la théorie, même sous l'aspect plus dynamique de sa tournure dialectique, préserve plus que jamais ses droits supérieurs, au point d'incorporer dans son royaume ce qui passe communément pour des expériences «pratiques» (le travail, le droit, la morale, l'éthicité, l'art...).

(3) L'attribution d'une position antireprésentationaliste à Hegel s'explique par la double focalisation qui vient d'être évoquée (et critiquée). S'il est notoire que Hegel mène une critique des philosophies qui absolutisent le «royaume de

25 Voir Hegel, introduction à la Phénoménologie de l'esprit, trad. B. Bourgeois (2006 [dorénavant abrévié Phéno.], p. 117-118).

26 Voir Olivier Tinland, Hegel. Maîtrise et servitude (2003, introduction, p. 22-26).

27 Sur ce point, voir Pierre-Jean Labarrière, Introduction à une lecture de la Phénoménologie de l'esprit de Hegel (1979, ch. VII). 
la représentation», une telle critique ne se veut nullement anti-représentationaliste, mais vise à marquer la supériorité du discours rationnel sur celui d'un entendement dont il présuppose constamment le travail fécond et indispensable ${ }^{28}$. L'activité rationnelle opère la décantation conceptuelle des représentations, leur dé-métaphorisation ${ }^{29}$, mais n'implique nullement une sortie radicale hors du «représentationalisme» : il s'agit bien plutôt de purifier les représentations de leurs scories sensibles, de les libérer de leur ancrage dans la subjectivité immédiate de l'esprit individuel et de fluidifier les oppositions figées que de telles scories génèrent dans la pensée d'entendement. S'il convient d'opérer une critique de la séparation abstraite du sujet et de l'objet, ainsi que d'une entente trop platement empiriste de la théorie correspondantiste de la vérité, ce n'est nullement pour renoncer à la dualité sujet-objet, ni à celle de correspondance, mais pour repenser celles-ci dans le cadre spéculatif d'une philosophie de l'esprit et du concept ${ }^{30}$. On ne saurait donc assimiler l'Aufhebung hégélienne de la Vorstellung à une remise en cause de la «spectator theory of knowledge» : pour le meilleur et pour le pire, l'idéalisme hégélien demeure une spéculation (qui certes n'est pas une «théorie» ordinaire), non un éloge de la pratique au détriment de la théorie ${ }^{31}$. Parler à son sujet d'antireprésentationalisme reviendrait à durcir en une discontinuité et en une opposition ce qui relève selon lui d'une élaboration dialectique continue ${ }^{32}$ :

28 Voir, dans la préface de la Phénoménologie de l'esprit, le célèbre éloge de l'entendement, «la puissance la plus étonnante et la plus grande, ou, bien plutôt, la puissance absolue» (Phéno., préface, p. 80). Voir aussi l'adage d'Iéna souvent cité : «La raison sans l'entendement n'est rien, l'entendement est pourtant quelque chose sans la raison. L'entendement ne peut être bradé» (Hegel, Notes et fragments. Iéna 1803-1806 [1991, §47, p. 63]).

29 Voir Hegel, Encyclopédie des sciences philosophiques, t. I. La science de la logique (1970, introduction, §3R, p. 166) : «les représentations en général peuvent être regardées comme des métaphores des pensées et des concepts». Nous citons ici la $3^{\mathrm{e}}$ édition de l'Encyclopédie (1830); dans la suite de l'article, nous employons l'abréviation Enc. 1830 - SL suivie de la pagination.

30 Voir par exemple Hegel, Science de la logique, livre troisième, Le concept (2016, p. 33) : la définition de la vérité comme correspondance «est d'une grande, et même, de la plus haute valeur».

31 Ce qui n'interdit pas, bien entendu, de souligner que la pensée a pour Hegel des enjeux pratiques décisifs : voir à ce sujet Franck Fischbach, «"Im Denken ist nichts Praktisches". Quelques remarques à propos d'une critique couramment adressée à Hegel», dans O. Tinland, dir., Lectures de Hegel (2005).

32 À cet égard, on pourrait se demander si Gérard Lebrun, dans sa présentation saisissante de l'originalité du «discours hégélien» (La patience du Concept. Essai sur le Discours hégélien [1972]), ne cède pas à la tentation de transformer ce qui n'est pour 
cela reviendrait à appliquer à un tel idéalisme une opposition d'entendement («ou bien — ou bien») dont il est le premier à remettre en cause la pertinence.

(4) Le rapprochement de Hegel et du pragmatisme suppose ici non seulement une sélectivité discutable des thèses et des textes hégéliens, mais aussi, symétriquement, une sélectivité tout aussi discutable des thèses représentatives du pragmatisme. Gimmler s'appuie ici essentiellement sur des thèmes qui proviennent des positions instrumentalistes de Dewey (relues avec des lunettes rortyennes pour en faire un «antireprésentationalisme») et assume ainsi d'emblée de ne considérer qu'une portion très congrue de l'ensemble du mouvement pragmatiste ${ }^{33}$. Cette double sélectivité, qui pourrait en soi avoir sa légitimité dans le cadre d'un dispositif méthodologique apte à en motiver l'adoption, devient un écueil dans le cadre d'un discours prétendant statuer de manière générale sur le «pragmatisme» de la «pensée hégélienne» : on perd alors sur tous les tableaux, puisque l'on s'interdit à la fois une perspective vraiment générale sur l'ensemble du pragmatisme et l'ensemble de la pensée de Hegel, et une perspective plus spécifique qui reviendrait à s'intéresser aux lectures effectives de cette pensée par tel ou tel grand représentant du pragmatisme. Ce n'est qu'en procédant à une double réduction, de l'idéalisme hégélien à une théorie «antireprésentationaliste» de la connaissance et du pragmatisme à une perspective instrumentaliste sur le rapport de la théorie à la pratique, qu'un recoupement des deux trajectoires devient vaguement plausible : si «Hegel» n'est plus vraiment Hegel, pas plus que le «pragmatisme» n'est vraiment le pragmatisme, les présupposés de départ finissent par miner le projet d'ensemble et le vider de sa substance. Il est donc nécessaire d'envisager une autre stratégie pour établir un rapport entre Hegel et le pragmatisme, qui soit compatible avec une interprétation moins sélective et moins partiale des deux termes du rapport.

\section{Une deuxième stratégie : la recherche d'un critère unique d'identification d'une perspective commune au pragmatisme et à Hegel}

Une manière d'échapper aux écueils de la première stratégie de rapprochement de Hegel et du pragmatisme consisterait à adopter une position plus englobante et à se demander, comme le fait Robert Stern, «s'il existe une perspective philosophique partagée par Hegel et les pragmatistes, ou si, sur un point crucial, ces positions sont vouées à se séparer» ${ }^{34}$. Selon Stern, c'est sur le plan

\section{(suite)}

Hegel qu'une dualité de niveaux de la pensée en un dualisme caractérisé par l'opposition entre l'entendement (la représentation) et la raison (le concept).

33 En particulier, Peirce, qui a pourtant fondé ce courant philosophique, et qui a proposé maintes réflexions critiques sur l'idéalisme hégélien, est le grand absent de la présentation du pragmatisme proposée par Gimmler.

34 Robert Stern, «Hegel and Pragmatism», reproduit dans Hegelian Metaphysics (2009, p. 210). Nous indiquons désormais la pagination dans le corps du texte. 
«épistémologique» qu'une telle question doit être posée en priorité, dans la mesure où c'est là «que se trouve le cœur de la perspective pragmatiste» $(\text { Stern, } 2009, \text { p. } 210)^{35}$. La première étape suppose donc (1) de déterminer ce que pourrait être une telle perspective (épistémologique) commune aux grands représentants du pragmatisme, après quoi la seconde étape consistera (2) à examiner la pensée hégélienne pour voir dans quelle mesure elle peut s'inscrire dans une telle perspective. Examinons successivement ces deux points.

(1) Une fois reconnue l'impossibilité «de réduire la perspective du pragmatisme à une simple formule», telle que la fameuse «maxime pragmatiste» ${ }^{36} \mathrm{de}$ Peirce ou ses variantes chez James, la recherche de Stern s'oriente vers un «réseau de croyances pragmatistes» afin d'en repérer le «centre», qu'il croit pouvoir identifier à une «épistémologie anticartésienne, dont on peut considérer que c'est à partir d'elle que le reste de la perspective pragmatiste se développe» (Stern, 2009, p. 210-211). Cette fois-ci, c'est non pas Dewey, mais Peirce, le père fondateur du pragmatisme, qui constitue le point de départ privilégié : on trouve en effet dans ses premiers grands articles des années 1860-1870 notamment «Quelques conséquences de quatre incapacités»» ${ }^{37}$ — une critique dévastatrice des grands piliers de la conception cartésienne de la connaissance que sont le recours préalable au doute méthodique et le rôle fondationnel dévolu à l'intuition. La première cible d'une telle critique est la «méthode cartésienne [qui] suppose que le fait que nous ne possédions pas une raison forte de croire à une proposition nous fournit une raison d'en douter» ${ }^{38}$ et qui généralise un tel présupposé sous la forme du doute méthodique. À l'idée

35 Nous ne discutons pas ici de la pertinence de cet argument, qui paraît particulièrement bien adapté à Peirce (une fois précisé que son épistémologie s'articule fortement à une métaphysique), mais qui pourrait être soumis à la critique en ce qui concerne les autres représentants du pragmatisme. Le cas extrême serait Rorty, qui, comme on le sait, définit le pragmatisme en rupture avec le projet de la «philosophie comme épistémologie»».

36 Voir Peirce, Collected Papers of C. S. Peirce (1931-1958, 5.402) : «Considérer les effets, dont il est concevable qu'ils aient des incidences pratiques [practical bearings], qu'a l'objet de notre conception tel que nous le concevons. Alors, notre conception de ces effets est la totalité de notre conception de l'objet». La version française originale (Revue philosophique, 7 janvier 1879) prenait une forme un peu plus simple : «Considérer quels sont les effets pratiques que nous pensons pouvoir être produits par l'objet de notre conception. La conception de tous ces effets est la conception complète de l'objet».

37 Traduit par $\mathrm{Cl}$. Tiercelin et $\mathrm{P}$. Thibaud dans Peirce, Pragmatisme et pragmaticisme. Euvres philosophiques, vol. 1 (Peirce, 2002).

38 Christopher Hookway, "'The Principle of Peirce' and the Origins of Pragmatism», dans A. Malachowski, dir., The Cambridge Companion to Pragmatism (2013, p. 22). 
d'un doute radical, hyperbolique et universel, Peirce oppose les réserves suivantes :

Nous ne saurions commencer par le doute complet. Il nous faut commencer avec tous les préjugés qui sont effectivement les nôtres quand nous abordons l'étude de la philosophie. Ces préjugés ne sauraient être dissipés par une maxime, car ce sont des choses dont il ne nous viendrait pas à l'esprit qu'elles puissent être mises en question. Ce scepticisme initial ne sera donc que duperie de soi, et non un doute réel; et aucun de ceux qui suivent la méthode cartésienne ne sera jamais satisfait qu'il n'ait auparavant recouvré toutes ces croyances qu'il a abandonnées pour la forme. C'est donc un préliminaire aussi inutile que d'aller au pôle Nord pour se rendre à Constantinople en descendant régulièrement le long d'un méridien. On peut, il est vrai, trouver au cours de ses recherches des raisons de douter de ce que l'on avait commencé par croire : mais en ce cas on doute parce qu'on a une raison positive de le faire, et non en tenant compte de la maxime cartésienne. Ne feignons pas de douter en philosophie de ce dont nous ne doutons pas en nos cœurs ${ }^{39}$.

Cette critique du doute méthodique (par opposition à un «doute réel», motivé par des «raisons positives») s'articule à une critique de l'intuitionnisme cartésien, qui sous-tend le privilège accordé au critère de la clarté et de la distinction des idées : un tel critère s'avère «insidieusement individualiste et immédiat» (Stern, 2009, p. 212), car il enferme le penseur dans le quant-à-soi de ses propres convictions (subjectivisme) au lieu d'élargir l'examen d'une idée à «la communauté des enquêteurs» ${ }^{40}$ (intersubjectivité). Ce rejet de l'intuitivité du critère de clarté et de distinction ${ }^{41}$ se prolonge dans la critique du fondationalisme cartésien — l'idée d'un unique ordre des raisons fondé sur des premiers principes indubitables — qui, tout en reposant sur l'intuitionnisme déjà critiqué, se trouve en porte-à-faux avec la méthode effective caractérisée par une approche plus faillibiliste et englobante — des «sciences qui ont connu des succès» : «ses raisonnements [de la philosophie] ne devraient pas prendre la forme d'une chaîne, laquelle ne sera jamais plus forte que son maillon le plus faible, mais d'un câble dont les fibres peuvent être

39 «Quelques conséquences de quatre incapacités» (Peirce, 2002, p. 38; traduction modifiée).

40 Stern, 2009. On trouve ailleurs d'autres arguments contre l'intuitionnisme, notamment celui de la régression à l'infini : pour savoir avec certitude qu'une connaissance est intuitive, il faudrait le savoir intuitivement (puisque seule l'intuition procure une certitude absolue), donc avoir une intuition de ce savoir intuitif de l'intuition, etc.

41 Voir Peirce, «Comment rendre nos idées claires» (2002, p. 240) : «La doctrine tant admirée des idées claires et distinctes, ce "joyau de la logique", est peut-être assez jolie, mais il est grand temps de reléguer au musée des curiosités cet antique bijou et de prendre quelque chose de plus assorti aux mœurs modernes». 
extrêmement fines, pourvu qu'elles soient suffisamment nombreuses et tissées serré» ${ }^{42}$. Un tel fondationalisme revient en outre à faire reposer l'édifice de la connaissance sur «un terme ultime absolument inexplicable et inanalysable» ${ }^{43}$ qui contredit l'impératif de «médiation» de son ordre des raisons. Peirce oppose ainsi à l'épistémologie cartésienne une conception concrète (on doute quand on a des raisons effectives de douter), discursive (l'intuition, pas plus que le sentiment ou la conviction, n'est un argument), collective (l'autorité épistémique vient de la communauté des chercheurs, pas de la conviction subjective d'un esprit solitaire), faillibiliste («Je n'admettrai pas [...] que nous connaissions quoi que ce soit avec une certitude absolue» [Peirce, 1931-1958, 7.108]) et antifondationaliste (il n'existe pas de principes premiers soustraits à l'examen, qu'ils soient connaissables ou inconnaissables) de la connaissance.

Selon Robert Stern, cette critique de la conception cartésienne de la connaissance constitue un socle commun à l'ensemble du pragmatisme, quoi qu'il en soit des différenciations et bifurcations qui ont scandé son développement ultérieur. En premier lieu, le rejet du doute méthodique au profit du «doute réel» conduit à une «image neurathienne de la connaissance») ${ }^{44}$ (Stern, 2009, p. 213) d'après laquelle le fait que toute croyance soit susceptible d'être remise en cause (faillibilisme) n'implique aucunement la nécessité de remettre toutes nos croyances en cause d'un seul coup, avant même d'avoir «quitté le port» (doute méthodique) : nous sommes voués à réparer notre bateau en pleine mer, planche par planche, dans un effort critique motivé par des obstacles réels, collectivement validé et conçu comme une tâche indéfinie. Comme on a pu le faire remarquer, une telle image «peut être considérée comme la conception qui se trouve au cœur des pensées de Peirce, James et Dewey» ${ }^{45}$ : il est également possible de retrouver cette idée chez certains «néopragmatistes» ${ }^{46}$.

La conception peircienne du «doute réel» se trouve aussi à la base d'une sensibilité typiquement pragmatiste à l'ancrage des pratiques de connaissance dans la vie concrète des individus et des groupes humains : l'incertitude est d'abord une question pratique, celle qui régit ce que Dewey nommera les «situations problématiques» en lesquelles s'amorcent les procédures d'enquête et de résolution des problèmes. Contrairement au doute cartésien, le pragmatisme souligne l'impact des questions théoriques sur les conduites concrètes et sur

42 Ibid.

43 Ibid., p. 38-39. Chez Descartes, c'est la volonté inscrutable de Dieu. Chez Kant, ce sera la «chose en soi», pensable mais inconnaissable.

44 Voir notamment Otto Neurath, «Énoncés protocolaires», dans A. Soulez, dir., Manifeste du Cercle de Vienne et autres écrits (2010, p. 211) : «Nous sommes tels des navigateurs obligés de reconstruire leur bateau en haute mer, sans jamais pouvoir le démonter dans un dock et le rebâtir à neuf avec de meilleures pièces».

45 Cheryl Misak, «Introduction», dans Ch. Misak, dir., New Pragmatists (2007, p. 4).

46 Voir par exemple Brandom, Tales of the Mighty Dead (2002, p. 116). 
la vie des individus - eux-mêmes appréhendés dans leur environnement naturel et social - aux prises avec des situations d'incertitude ${ }^{47}$.

Dans la continuité de Peirce, les représentants du pragmatisme soulignent en outre la nécessité de s'intéresser à la pratique effective des sciences de la nature et au rôle précis qu'y joue le doute dans le cadre intersubjectif et social des communautés de chercheurs. Face au scepticisme solitaire, artificiel et abstrait de l'auteur des Méditations métaphysiques, il s'agit de promouvoir un faillibilisme collectif, ancré dans les pratiques réelles de la recherche théorique et motivé par les obstacles réels que celles-ci rencontrent. Cela vaut aussi, et d'abord, de la métaphysique, qui doit elle-même prendre ses distances vis-à-vis de «l'esprit de théologie» et adopter une démarche résolument expérimentale et faillibiliste analogue à celle des sciences de la nature (selon Peirce $)^{48}$, quand elle ne doit pas être abandonnée purement et simplement, et avec elle l'ambition anachronique d'une prima philosophia surplombant les autres secteurs de la culture (selon Dewey et Rorty) ${ }^{49}$. Il s'agit alors d'opérer une désolidarisation de l'entreprise du doute cartésien d'avec son arrière-plan métaphysique : coupé de son objectif théorique initial (la quête d'une certitude absolue et définitive, séparée des contextes effectifs d'enquête), le doute peut

47 Le contraste suggéré ici par Stern est sans doute exagéré : pour Descartes, la philosophie «signifie l'étude de la sagesse, [...] une parfaite connaissance de toutes les choses que l'homme peut savoir, tant pour la conduite de sa vie que pour la conservation de sa santé et l'invention de tous les arts» (Lettre-préface aux Principes de la philosophie). Il insiste ensuite sur «l'utilité de la philosophie»: «chaque nation est d'autant plus civilisée et polie que les hommes y philosophent mieux; et ainsi [...] c'est le plus grand bien qui puisse être en un État que d'avoir de vrais philosophes» (ibid.). Ce qui vaut de l'État vaut de l'individu : «c'est proprement avoir les yeux fermés, sans tâcher jamais de les ouvrir, que de vivre sans philosopher» et «cette étude est plus nécessaire pour régler nos mœurs et nous conduire en cette vie, que n'est l'usage de nos yeux pour guider nos pas» (ibid.). Quant aux sciences, elles nous permettent de nous rendre «comme maîtres et possesseurs de la nature» et d'œuvrer à la «conservation de la santé, laquelle est sans doute le premier et le fondement de tous les autres biens de cette vie» (Discours de la méthode, $6^{\mathrm{e}}$ partie). La portée pratique de la connaissance est donc constamment soulignée. Pour autant, le contexte de l'activité théorique, notamment sous la forme de la prima philosophia, est nettement distingué du contexte de la pratique : le doute méthodique trouve sa place dans le contexte théorique, mais il est incompatible avec les exigences de l'action, dont la temporalité contrainte («l'action ne souffre aucun délai») nous voue à nous fier à ce qui n'est que vraisemblable (Principes de la philosophie, $1^{\text {ère }}$ partie, $\left.\S 3\right)$. Voir Peirce, «Scientific Metaphysics», dans Collected Papers, vol. VI (1935, préface).

49 De ce point de vue, Brandom tient une forme d'équilibre précaire entre la métaphysique faillibiliste de Peirce et le renoncement rortyen à la métaphysique. 
être rendu aux pratiques contextuelles et évolutives de la recherche (et à sa quête de certitudes relatives et provisoires).

En dépit de la variété des manières d'en exploiter les potentialités, les grands gestes «anticartésiens» de Peirce paraissent constituer un noyau commun à l'ensemble de la tradition pragmatiste. Il semble donc envisageable de se servir d'une telle «épistémologie anticartésienne» comme critère de base permettant d'assimiler une philosophie au pragmatisme : à cet égard, il paraît opportun de s'interroger sur le «pragmatisme» de Hegel, ce qui suppose désormais de se demander si sa propre conception de la connaissance peut être considérée comme «anticartésienne», selon des modalités sinon identiques, du moins analogues à celles que proposait Peirce dans ses premiers grands textes sur la nature de la connaissance. En deçà des ressemblances partielles avec tel ou tel penseur pragmatiste qu'il est possible de repérer (le réalisme conceptuel, qu'il partage pour partie avec Peirce, la critique des dualismes abstraits, que l'on retrouve chez Dewey, l'historicisation des formes de la culture, abondamment thématisée par Rorty), en deçà aussi des divergences de vues parfois considérables qui se sont faites jour dans l'histoire du pragmatisme ${ }^{50}$, il serait possible de disposer d'un point de recoupement plus large, d'un engagement théorique fondamental et commun, qui concerne l'ensemble des représentants du pragmatisme classique et contemporain. Il deviendrait alors secondaire de s'interroger sur les nombreux thèmes (à commencer par celui de «l'esprit absolu») qui paraissent de nature à exclure d'emblée l'auteur de la Phénoménologie de l'esprit d'une telle tradition : la remontée vers un critère commun de définition du pragmatisme laisse entrevoir la possibilité d'une caractérisation primaire de Hegel comme étant (ou n'étant pas) pragmatiste.

(2) Une étape décisive de l'argumentation de Stern consiste dès lors à s'interroger sur la pertinence des interprétations de l'idéalisme hégélien qui reconduisent celui-ci à une forme de stratégie philosophique «cartésienne» (une telle manière de lire Hegel étant incarnée selon l'auteur, à tort ou à raison, par les commentaires de Stephen Houlgate ${ }^{51}$ ) et semble à ce titre invalider par avance

50 Pour prendre la mesure de telles divergences, voir H.O. Mounce, The Two Pragmatisms: from Peirce to Rorty (1997). L'auteur n'inclut pas dans son étude les représentants actuels du néopragmatisme (Brandom, Price), ce qui aurait contribué à accentuer encore davantage le caractère proliférant de telles divergences, dont on pourra trouver une illustration divertissante dans le collage de citations imaginé par Susan Haack, «"We Pragmatists...": Peirce and Rorty in Conversation», dans Manifesto of a Passionate Moderate. Unfashionable Essays (1998).

51 Robert Stern prend principalement appui sur deux ouvrages de Stephen Houlgate : An Introduction to Hegel: Freedom, Truth and History (2005) et The Opening of Hegel's 'Logic' (2006). Il peut s'appuyer sur des formules telles que celle-ci : «Le chemin du "doute universel" qui conduit dans la Science de la logique de Hegel 
une telle stratégie de rapprochement entre Hegel et le pragmatisme. Cette lecture «cartésianisante» de Hegel suppose de mettre en exergue les passages où celui-ci exige une «complète absence de présupposition» de la science philosophique au moment où celle-ci s'instaure en son commencement :

Tous les [...] présupposés ou préjugés sont à abandonner quand on entre dans la science, qu'ils soient empruntés à la représentation ou à la pensée; car c'est dans la science seulement que sont examinées toutes les déterminations de ce genre et que doit être reconnu ce qui est en elles et dans leurs oppositions. [...] La science devrait être précédée par le doute à l'égard de tout, c'est-à-dire par l'entière absence de présupposition en tout ${ }^{52}$.

Un passage de ce genre laisserait entendre, du point de vue de Stephen Houlgate, que Hegel reprend (sous une forme différente et avec des objectifs différents ${ }^{53}$ ) l'exigence cartésienne de libérer la science rationnelle de tout

(suite)

ressemble de toute évidence beaucoup à celui pris par Descartes» (Houlgate, 2006, p. 31). Ou sur celle-là : «Ce que Hegel apprend de Descartes et de Kant est que la pensée humaine nous libère de l'autorité arbitraire en soumettant toutes choses à l'examen minutieux de la raison qui s'autodétermine» (Houlgate, 2005, p. 27). Ou encore : «[Hegel] nous demande de suivre Descartes et de suspendre notre jugement au sujet de ce que nous avons auparavant considéré comme allant de soi, jusqu'à ce qu'ait été trouvée une manière de montrer si nos modes de pensée traditionnels sont justifiés» (ibid., p. 30). Ou enfin : «La meilleure manière de comprendre Hegel est de le considérer comme illustrant la volonté cartésienne de suspendre ses croyances et ses habitudes de pensée les plus chères, et de n'accepter comme vrai que ce que la raison elle-même détermine comme étant vrai» (ibid., p. 39). Enc. 1830 - SL, §78, p. 342. Voir aussi Leçons sur la logique. Berlin 1831, trad. J.-M. Buée et D. Wittmann (2007, p. 88) : «Il faut abandonner toutes les présuppositions [...]. Déjà Descartes a dit : De omnibus dubitandum est, il faut commencer par le doute ou, à proprement parler, par le désespoir». Hegel précise ensuite ses réserves à l'égard de la notion de doute, sur lesquelles il faudra revenir : «Le doute exprime davantage une approche extérieure, une découverte de la contradiction et de l'inquiétude de la contradiction, à dire vrai cette inquiétude et cette contradiction sont ce qui pousse à la philosophie» (ibid.). L'incarnation philosophique d'un tel désespoir qui est ensuite présentée par Hegel n'est pas le doute cartésien, mais le scepticisme antique.

53 Voir Houlgate, The Opening of Hegel's Logic (2006, p. 31-32) : «Le chemin du "doute universel" qui conduit à la Science de la logique de Hegel ressemble manifestement beaucoup à celui pris par Descartes. Cependant, la conclusion de Hegel n'est pas “je pense, donc je suis" mais plutôt "penser, donc est [thinking, therefore is]"». 
présupposé par la remise en cause de tout ce que celle-ci pourrait considérer comme acquis. La mise à l'écart de tout présupposé doit dégager le terrain pour la présentation de l'être pur, qui lance l'examen dialectique des catégories dans la Science de la logique. Aucun contenu déterminé ne saurait ici interférer avec la thématisation minimale de l'être, pas plus qu'une quelconque méthode préétablie et arbitrairement imposée de l'extérieur au «contenu» logique : il s'agit de se donner l'être pur, de «regarder faire [look on]» et de «voir ce qui se passe [see what happens]» (Stern, 2009, p. 217) ${ }^{54}$. Dans une telle perspective, le projet hégélien est interprété comme «complétant [le] projet kantien d'une autocritique radicale, que Kant lui-même a échoué à accomplir dans la mesure où il tenait trop de choses pour acquises concernant la nature de la pensée et des catégories» (ibid., p. 217), et indirectement comme complétant le projet cartésien d'une «libération de la conscience humaine» (ibid.) dont le criticisme kantien constituait déjà un premier prolongement. Hegel accomplirait ainsi le projet de la modernité, celui de l'élaboration «d'une pensée autocritique» en vue de fonder la connaissance scientifique, mais aussi «la liberté et la dignité humaines» (ibid., p. 218) : en poussant un tel projet jusqu'à ses plus extrêmes limites, il apparaîtrait donc comme la «quintessence du philosophe moderne» ${ }^{55}$.

Pour peu que l'on accepte les conclusions d'une telle lecture, de l'aveu de Stern, l'idée de rattacher Hegel au pragmatisme — selon le critère précité — paraît «totalement erronée, en dépit des ressemblances superficielles que l'on peut trouver entre eux» $(2009$, p. 218). Pour autant, si les textes qui portent sur le commencement de la Logique peuvent rendre plausible une lecture «cartésienne» de Hegel, d'autres textes permettent de tirer des conclusions opposées. C'est notamment le cas - une fois encore - de l'introduction à la Phénoménologie de l'esprit, en laquelle s'expose une argumentation «très proche de la distinction peircienne entre le doute réel et le doute artificiel», qui peut servir de pierre de touche à une présentation «anticartésienne» de la philosophie hégélienne. Stern mobilise le début de l'introduction, dans lequel Hegel prend pour cible une «représentation naturelle» ${ }^{56}$ de la connaissance (que l'on peut rattacher à Kant et à Reinhold, mais aussi, dans une certaine mesure, à Descartes et à Locke) selon laquelle il serait nécessaire, antérieurement à la mise en œuvre de toute connaissance déterminée, de procéder à un examen préalable des modalités qui rendent celle-ci possible, afin de se prémunir par avance contre «les nuées de l'erreur» là où l'on entend atteindre «le ciel de la vérité» ${ }^{57}$. À l'instar de l'auteur de l'Essai sur

\footnotetext{
54 Voir aussi ibid., p. 60 : «Nous devons simplement assister [attend] à la pensée de l'être pur et suivre son auto-transformation immanente.»

55 L'expression est de Houlgate (2006, p. 39).

56 Phéno., introduction, p. 117.

57 Ibid., p. 117.
} 
l'entendement humain et de celui de la Critique de la raison pure, on veut «examiner la faculté de connaître avant de connaître», donc «on veut connaître déjà avant de connaître», nonobstant le fait que «cet examen est lui-même déjà un acte de connaissance»» ${ }^{58}$. D'une telle représentation découle l'assimilation de la connaissance à un «instrument», un «intermédiaire», un «outil» ou un «milieu passif», donc à un «moyen» ${ }^{59}$ qui se tient entre le sujet connaissant et son objet (l'absolu), et dont il convient d'inspecter la nature propre avant de se mettre en quête d'une connaissance effective de l'absolu. Selon Hegel, le mal est ici dans le remède : en se représentant que «l'absolu se tiendrait d'un côté et que, de l'autre côté, la connaissance [...] qui, en tant qu'elle est en dehors de l'absolu, est bien aussi en dehors de la vérité, serait pourtant vraie», on cède à une «supposition par laquelle ce qui s'appelle crainte de l'erreur se fait connaître comme crainte de la vérité» ${ }^{60}$. Pour examiner mes capacités cognitives, je dois déjà connaître, et je fais alors face à un dilemme : ou bien ces capacités sont fiables et l'examen préalable s'avère dès lors superflu, ou bien elles ne le sont pas et l'examen lui-même est rendu impossible, faute d'un instrument valide pour évaluer la validité théorique de cet instrument.

Une telle prudence méthodologique est donc infructueuse, mais elle est aussi déplacée, car elle ne correspond pas à la manière dont l'enquête rationnelle procède effectivement : plutôt que de «se tourmenter avec [c]es échappatoires», la science peut «s'épargner la peine de prêter quelque attention que ce soit à de telles représentations et manières de parler par lesquelles la science elle-même doit être écartée» ${ }^{61}$. Selon Stern, un tel argument est typiquement «peircien» (2009, p. 220) : tandis que Descartes cède à ce que Hume nommait l'«antecedent scepticism» ${ }^{62}$, Hegel enjoint à la science de se mettre

58 Enc. 1830 - SL, Add. 1 §41, p. 497.

59 Phéno., introduction, p. 117-118.

60 Ibid., p. 119.

61 Ibid., p. 120.

62 Voir Hume, Enquête sur l'entendement humain, dans Essais et traités sur plusieurs sujets, trad. M. Malherbe (2004, section XII, p. 173) : «Il y a une espèce de scepticisme, antérieur à l'étude et à la philosophie, qui a été fort recommandé par Descartes et d'autres philosophes, comme un préservatif souverain contre l'erreur et la précipitation du jugement. Il prône un doute universel, appliqué non seulement à toutes nos opinions et tous nos principes antérieurs, mais aussi à nos facultés mêmes : nous devons, disent-ils, nous assurer de leur véracité par une chaîne de raisonnements, déduite d'un principe premier qui ne puisse être ni fallacieux ni trompeur. Mais, d'une part, il n'y a pas de principe premier qui aurait une telle prérogative sur les autres principes qui sont immédiatement évidents et convaincants; d'autre part, quand un tel principe existerait, nous ne pourrions avancer d'un pas sinon par l'usage de ces facultés dont nous sommes censés nous défier entièrement. Le doute cartésien, si quelque être humain pouvait y parvenir (ce qui manifestement 
en œuvre sans préalables inutiles et d'affronter non pas les «doutes» abstraits de la réflexion méthodologique, mais l'équivalent phénoménologique du «consequent scepticism» de Hume, à savoir le «désespoir» réel des contradictions rencontrées en chemin ${ }^{63}$. Là où le doute ne fait qu' «ébranler telle ou telle vérité présumée», le désespoir est le «scepticisme en train de s'accomplir» et à ce titre, il ne saurait se réduire au «propos de ne pas, dans la science, s'en remettre, sur autorité, aux pensées d'autrui, mais de tout examiner par soi-même et de ne suivre que sa conviction propre, ou mieux encore : de tout produire soi-même et de ne tenir pour le vrai que ce que l'on fait soi-même» $^{64}$. Il est un "scepticisme qui se dirige sur tout le champ de la conscience prise en son apparaître» et qui «amène à désespérer des représentations, pensées et opinions prétendument naturelles [...] et dont la conscience qui se met directement à examiner est encore remplie et affectée»» $^{65}$ : le désespoir phénoménologique est l'épreuve effective d'un cheminement épistémique en train de se faire, non l'examen préalable d'un cheminement possible. En croyant se libérer de toute présupposition par un tel examen, le philosophe (cartésien, lockien, kantien, reinholdien) demeure sous l'emprise d'une présupposition soustraite à l'examen, celle de la connaissance conçue comme un instrument (ou intermédiaire) qu'il faut soumettre à une critique préalable de ses capacités. Il cède par ailleurs à l'inconséquence de mettre en œuvre une connaissance (soustraite à l'examen) pour mener l'examen critique de ses pouvoirs cognitifs (ce qui conduit soit à une régression à l'infini dans l'examen desdits pouvoirs, soit à une pétition de principe qui ne dit pas son nom). Aux doutes abstraits et provisoires du cartésianisme, il faut donc substituer la mise à l'épreuve effective des prétentions épistémiques de la conscience dont la Phénoménologie de l'esprit se veut la présentation immanente.

On peut donc trouver dans une telle critique de la manière dont certaines philosophies conçoivent l'examen préalable de la possibilité de connaître l'équivalent du contraste peircien entre «doute artificiel» et «doute réel»: contrairement au doute cartésien, les doutes de la conscience aux prises avec les contradictions de ses propres présupposés épistémiques (qui s’objectivent dans son expérience) sont des doutes motivés, concrets, liés à des configurations précises du savoir en proie à ses propres contradictions. Face au «questionnement infondé de nos croyances» incarné dans le doute cartésien (Stern, 2009,

(suite)

n'est pas le cas), serait donc entièrement incurable; et aucun raisonnement ne pourrait nous ramener à un état d'assurance et de conviction sur aucun sujet».

63 À cette distinction correspond le contraste entre Zweifel et Verzweiflung : «[un tel chemin] peut [...] être regardé comme le chemin du doute, ou, plus proprement, comme le chemin du désespoir» (Hegel, Phéno., introduction, p. 122).

64

Ibid.

65 Ibid., p. 123. 
p. 225), il s'agit de proposer des «raisons réelles» de douter : il semble donc possible de dégager, à partir de telles considérations, un «terrain commun à Hegel et au pragmatisme». Mais cela suppose d'invalider les lectures «cartésiennes» précédemment évoquées, en particulier leur interprétation de l'exigence hégélienne de «l'absence de tout présupposé» comme se situant sur le même plan que le doute méthodique de Descartes.

En s'appuyant sur des analyses de John Skorupski ${ }^{66}$, Stern opère une distinction décisive entre deux conceptions de la «liberté» de la pensée susceptibles de soustendre les projets philosophiques de la modernité. La première, identifiée à «l'idée cartésienne», est censée se prolonger dans l'ensemble de la philosophie allemande moderne, de Kant à Hegel : il s'agit d'un examen de la pensée par elle-même, de ses possibilités, de ses limites et de ses présuppositions. La seconde, nommée «la pensée de l'intérieur [thinking from within]», illustrée notamment par John Stuart Mill, consiste non pas à refuser tout présupposé (ce qui est impossible), mais à adopter une «ouverture d'esprit critique permanente au sujet de tout ce que nous estimons savoir» ${ }^{67}$, dans une optique naturaliste (le sujet de la connaissance fait partie du monde qu'il connaît), holiste (le travail critique s'opère au sein de la totalité de nos convictions) et faillibiliste (rien n'est par principe soustrait à la critique) : c'est ici non pas l'absence de présuppositions, mais l'absence de contraintes (ou de limites arbitraires à l'autocritique permanente de la pensée) qui joue le rôle de critère de la liberté de penser. Le pragmatisme, par son faillibilisme critique proclamé, s'inscrit clairement dans cette lignée. Qu'en est-il de Hegel? Selon Skorupski, si l'exigence de l'absence de présupposés rattache celui-ci à la première tradition (cartésienne), néanmoins «la méthode de Hegel, soit dit en passant, pourrait aussi être décrite comme une pensée de l'intérieur» ${ }^{68}$ dans la mesure où elle consiste à partir d'une situation socio-historique déterminée du penseur («chacun est de toutes façons un fils de son temps» ${ }^{69}$ ) et à élaborer une critique immanente d'un tel contexte ${ }^{70}$.

Cette ambiguiité de la conception hégélienne de la liberté de penser incite à préciser le sens de son exigence de renoncer à tout présupposé : selon Stern, les «présuppositions» que Hegel prend pour cible ne sont pas des croyances

66 John Skorupski, Why Read Mill Today? (2006, p. 6 sqq).

67 Ibid., p. 8.

68 Ibid., p. 9.

69 Hegel, Principes de la philosophie du droit, trad. J.-F. Kervégan (2013, Préface, p. 132).

70 Une telle critique immanente du contexte historique du penseur est le trait que Rorty a considéré comme constituant l'apport fondamental de Hegel au pragmatisme : «Hegel ne pense pas que la philosophie puisse s'élever au-dessus des pratiques sociales de son temps, pour juger de leur opportunité [desirability], vers quelque chose qui ne soit pas soi-même une pratique sociale alternative (passée ou future, réelle ou imaginée)» (Rorty, Philosophy as Cultural Politics [2007, p. 23]). 
présentes dans l'individu, mais des choses qui sont «posées avant» la pensée et qui ne peuvent être «saisies ni comprises» (Stern, 2009, p. 228), à l'instar du Dieu de Jacobi, sans recourir à des moyens extra-discursifs («positifs») d'établissement de l'autorité épistémique (intuition ou révélation). En ce sens, il s'agit pour Hegel de refuser toute présupposition externe, susceptible de contraindre l'exercice de la libre pensée de l'extérieur du champ rationnel et de l'empêcher à ce titre d'être «chez elle [bei sich]», donc libre. En réalité, selon Stern, l'exigence de renoncer à tout présupposé concerne un domaine particulier de la philosophie hégélienne : le commencement de la logique. Dans un tel cadre, il existe des motivations spécifiques (des «raisons positives», dirait Peirce) permettant de justifier un tel renoncement :

1) contrairement aux autres sciences, la logique ne peut présupposer aucune méthode de pensée, puisque celle-ci fait partie de son objet de recherche ${ }^{71}$;

2) contrairement aux autres sciences, la logique ne peut présupposer aucune représentation de son objet, dans la mesure où la pensée (conceptuelle) excède le registre de la Vorstellung ${ }^{72}$;

3) là où les sciences empiriques opèrent avec des procédés contingents, la logique doit mettre en évidence la nécessité de son propre déploiement, donc ne pas être tributaire de présuppositions qui ne font pas l'objet d'une déduction rationnelle (ce que l'on pourrait appeler la «clause fichtéenne» de la logique) $)^{73}$.

71 Voir Enc. 1830 - SL, Introduction, §1, p. 163 : «La philosophie est privée de l'avantage dont profitent les autres sciences, de pouvoir présupposer ses $o b$-jets, comme accordés immédiatement par la représentation, ainsi que la méthode de la connaissance pour commencer et pour progresser comme déjà admise». Voir aussi Science de la logique, livre premier, L'être, trad. B. Bourgeois (Hegel, 2015, introduction, p. 49) : «La logique [...] ne peut présupposer aucune de ces formes de la réflexion ou de ces règles et lois de la pensée, car elles constituent une partie de son contenu et elles ont à être fondées au préalable à l'intérieur d'elle». Le livre premier de Science de la logique est dorénavant abrévié SL 1.

72 Voir Enc. 1830 - SL, Introduction, \$17, p. 183 : «Pour ce qui est du commencement que la philosophie a à instaurer, elle semble en général commencer avec une présupposition subjective, comme les autres sciences, c'est-à-dire être contrainte de faire d'un ob-jet particulier tout comme c'est ailleurs l'espace, le nombre, etc., ici, c'est la pensée l'ob-jet de sa pensée. Mais c'est l'acte libre de la pensée que de se placer au point de vue où elle est pour elle-même et en cela se crée et se donne elle-même son ob-jet».

73 Voir Enc. 1830-SL., §9, p. 173 : «la raison subjective demande à être aussi satisfaite suivant la forme; cette forme est la nécessité en général. [...] Dans ce type de science 
Le refus de toute présupposition est donc lié ici non pas à la nature générale de l'activité philosophique ${ }^{74}$, mais à la nature spécifique du projet de la science de la logique, qui prend pour objet de sa recherche ce qui constitue habituellement un présupposé de la recherche (méthode de la science, représentations préalables de l'objet de la science, représentations considérées comme des points de départ absolus ou des «principes» de la science).

Pour user d'un vocable peircien, un tel refus renvoie non pas à un «doute artificiel», mais à un «doute réel», motivé par des problèmes précis liés à l'originalité du projet de la Science de la logique : c'est précisément le rôle de la Phénoménologie de l'esprit, conçue comme une introduction critique au «système de la science», que de mettre en scène les «doutes réels» qui surviennent à la faveur de l'épreuve des contradictions des présupposés épistémiques de la conscience puis de dégager les conditions d'un «savoir absolu», «délié» de telles présuppositions qui font barrage à l'investigation immanente de la pensée libre ${ }^{75}$. On comprend alors que «l'exigence d'un tel scepticisme accompli», qui requiert «l'entière absence de présupposition en tout», se traduise par «la résolution de vouloir purement penser, grâce à la liberté qui fait abstraction de tout et saisit sa pure abstraction, la simplicité de la pensée»» ${ }^{76}$ : une telle liberté n'est pas celle, inutile et infructueuse, du doute cartésien (qui n'en constitue qu'une préfiguration imparfaite), mais la mise en œuvre d'un scepticisme radical à l'égard des présupposés qui viennent faire obstacle à la mise en œuvre strictement immanente de la «pensée pure». Cette exigence, loin d'éloigner Hegel du pragmatisme (en le rapprochant du cartésianisme), semble au contraire répondre à des raisons précises, motivées, «réelles» de douter, et le rapprocher à ce titre de la conception peircienne de l'enquête scientifique.

On pourrait néanmoins se demander, comme le fait Stern (2009, p. 234), si l'exigence hégélienne de refuser tout présupposé à la recherche logique, quand bien même elle serait motivée par des obstacles spécifiques à la mise en œuvre de ce projet, ne garde pas une allure fondamentalement cartésiano-kantienne (donc anti-pragmatiste), par le seul fait d'estimer

(suite)

dont on a parlé plus haut [les sciences positives], [...] les commencements sont toutes sortes d'immédiatetés, d'être-trouvé, de présuppositions. Dans [ce] cas, satisfaction n'est pas donnée à la forme de la nécessité».

74 Ainsi, au niveau de la philosophie de la nature, «la naissance et formation de la science philosophique a la physique empirique pour présupposition et condition» (Encyclopédie des sciences philosophiques, t. II, Philosophie de la nature, trad. B. Bourgeois [2004, p. 186]). Le rejet de toute présupposition ne concerne donc pas la Realphilosophie, qui doit nécessairement s'échafauder à partir des représentations et des procédures théoriques des sciences empiriques.

Sur ce point, voir $S L$ 1, Introduction, p. $55 \mathrm{sq}$.

Enc. $1830-S L, \S 78 \mathrm{R}$, p. 342. 
réalisable une telle libération à l'égard de tout présupposé : n'entre-t-elle pas ici en contradiction avec l'injonction peircienne à «commencer avec tous les préjugés que nous avons effectivement»? Faute de remettre en cause la légitimité de l'exigence de renoncer à tout présupposé, ne faut-il pas à tout le moins, d'un point de vue pragmatiste, remettre en cause la croyance en la faisabilité d'un tel renoncement? Une telle exigence ne risque-t-elle pas de «bloquer le chemin de l'enquête» en rendant impossible toute progression théorique, faute de point d'appui disponible ${ }^{77}$ ? Quoi qu'il en soit, l'essentiel de la démonstration demeurerait valide : Hegel partagerait avec le pragmatisme une conception anticartésienne de l'épistémologie et son exigence de renoncer à tout présupposé dans l'abord de la logique serait, tout bien considéré, compatible avec une telle épistémologie, quand bien même la question de la possibilité de réaliser une telle mise entre parenthèses de tout présupposé pourrait constituer une pomme de discorde avec certains représentants du pragmatisme.

À présent, comment évaluer la pertinence d'une telle stratégie de rapprochement de Hegel et du pragmatisme? En premier lieu, il est patent qu'elle constitue un procédé beaucoup plus convaincant que le précédent (illustré par l'article de Gimmler), dans la mesure où elle suppose de se rapporter à un socle commun de thèses partagées par l'ensemble (ou peu s'en faut) des représentants du pragmatisme, plutôt que de mobiliser de manière arbitraire certaines caractéristiques isolées de certaines versions de ce mouvement (Dewey, Rorty). En outre, sur l'autre versant, cette stratégie permet d'exploiter non pas seulement certains textes isolés de Hegel (eux-mêmes tirés d'une seule œuvre, censée représenter l'ensemble de la philosophie hégélienne alors qu'elle n'en est que l'introduction : la Phénoménologie de l'esprit) en absolutisant de manière illégitime leur portée (relative), mais un réseau beaucoup plus étendu de textes fondamentaux permettant d'affermir l'hypothèse de départ. En sa forme, la démarche de Stern semble donc beaucoup plus légitime, dans la mesure où elle articule ce qui est commun aux pragmatistes (en deçà de leurs différences doctrinales) et la pensée hégélienne envisagée dans son ensemble (au-delà du contenu apparent de tel ou tel aspect isolé de celle-ci).

Que penser, par ailleurs, de son contenu? Sur ce plan, les résultats de cette démarche s'avèrent — de l'aveu même de l'auteur — bien modestes : la mise en évidence d'une «épistémologie anticartésienne» à certains égards commune à Hegel et au pragmatisme constitue une thèse qui est somme toute de faible portée, dans la mesure où il est patent qu'il existe une immense variété de manières d'être «anticartésien». Il ne serait sans doute pas impossible de

77 Comme le fait remarquer Robert Stern à la suite de Christopher Hookway, il est possible que Peirce lui-même juge une telle absence de présupposition nécessaire pour la conduite de certains projets théoriques, notamment l'élaboration de sa propre sémiotique. 
démontrer que ce qui vaut ici de Hegel vaut aussi bien de l'ensemble des philosophes qui ont suivi Descartes (pour ne pas parler de ceux qui l'ont précédé ${ }^{78}$ ): déjà, Gassendi, Hobbes, Leibniz ${ }^{79}$, Spinoza et Malebranche se sont nettement démarqués de la conception cartésienne de la connaissance, et l'empirisme moderne (Locke, Berkeley, Hume), le kantisme et les diverses formes de postkantisme se sont élaborés dans une constante opposition à Descartes et à sa conception du doute, de l'intuition, de la méthode, du cogito, de la liberté, des critères de vérité, des rapports de l'âme et du corps, de Dieu, etc. Voudrait-on préciser la perspective et n'entendre par «épistémologie cartésienne» qu'une définition de la connaissance par le rôle fondationaliste dévolu à l'intuition et le rôle méthodiquement préliminaire dévolu au doute universel, là encore il serait aisé d'inclure la quasi-totalité des successeurs de Descartes dans la catégorie des promoteurs d'une «épistémologie anticartésienne». Un tel critère de démarcation est si ténu qu'il s'avère impropre à établir une authentique ligne de séparation entre les «cartésiens» et les «anticartésiens», dans la mesure où le seul «cartésien» authentique de l'histoire de la philosophie (y compris en ramenant le cartésianisme au rôle dévolu à l'intuition et au doute méthodique) n'est autre que Descartes lui-même. On peut certes tenter de proposer (comme le fait Skorupski) des regroupements en lignées plus englobantes, et distinguer par ce biais différentes conceptions de la liberté de penser : mais là encore, Hegel, dans sa tentative de «penser de l'intérieur», se retrouve accompagné de tant d'autres penseurs par ailleurs si différents de lui (Mill!) que l'on en vient à douter de l'opportunité de tels regroupements.

Que la pensée de Hegel se démarque de l'«épistémologie cartésienne», c'est ce que ses leçons sur la philosophie de Descartes permettent de confirmer aisément ${ }^{80}$. Mais faute de spécifier les raisons d'une telle démarcation (qui

78 L'originalité de Peirce sera précisément d'inverser la lecture téléologique de l'histoire de la philosophie classique en montrant la supériorité, à certains égards, de la pensée scolastique sur celle de Descartes, contrairement à l'image (cartésienne) reçue.

On se contentera ici de mentionner l'ouvrage d'Yvon Belaval, Leibniz critique de Descartes (1978).

80 Voir en particulier Leçons sur l'histoire de la philosophie, t. VI, trad. P. Garniron (1985, chapitre «Descartes», p. 1389), où le projet cartésien du doute méthodique est d'emblée considéré comme rendu nul et non avenu en raison de l'ancrage cartésien dans la pensée d'entendement : Descartes «partit de l'idée qu'il faut écarter toute présupposition, que la pensée doit commencer par elle-même [...]. Mais comme le penser ne s'est alors saisi à proprement parler que comme entendement abstrait, les représentations déterminées, le contenu ne furent pas dérivées de l'entendement, mais accueillis de manière empirique». Hegel s'en prend ensuite aux «arguments populaires» qui motivent ce doute (ibid., p. 1394) et à l'absence de toute justification rigoureuse de ce procédé : «nous ne devons pas tenir la 
s'avèrent bien différentes chez Hegel et chez Peirce : pour Hegel, c'est d'abord l'adhésion de Descartes à une pensée d'entendement rebelle à toute dialectisation qui voue le doute cartésien à l'ineffectivité des «commencements vides» ${ }^{81}$ ), les arguments pour en inférer une forme de «pragmatisme» hégélien s'avèrent beaucoup trop indéterminés pour emporter l'adhésion. On peut sans doute s'en tenir à une stratégie purement défensive - comme le fait Stern lui-même - consistant à montrer que la conception hégélienne de la connaissance n'est pas rigoureusement incompatible avec le socle «anticartésien» de l'épistémologie pragmatiste : mais une telle stratégie de repli ne permettra pas de démontrer grand-chose, si ce n'est que Hegel, tout bien considéré, et tout comme l'ensemble des innombrables successeurs de l'auteur des Méditations métaphysiques, n'est pas cartésien. Cela ne suffit pas, loin s'en faut, à en faire un pragmatiste.

\section{Remarques conclusives}

Nous faisons donc face à un double constat d'échec : l'approche thématique a montré ses limites, qui consistent pour l'essentiel dans une sélectivité très discutable des thèses et des textes mobilisés pour faire se rejoindre, de manière quelque peu forcée, les deux côtés du rapport «Hegel/pragmatisme» autour d'intitulés qui sont au mieux approximatifs (primat de la pratique), au pire totalement inadaptés (antireprésentationalisme). De son côté, l'approche critérielle, tout en évitant salutairement de tels écueils et en faisant droit à une prise en compte beaucoup plus large des textes hégéliens et des différentes tendances du pragmatisme, doit s'en tenir à une définition minimaliste de son point de recoupement (le rejet du «doute artificiel»), ce qui implique un usage trop peu discriminant de la distinction entre les positions «cartésienne» et «anticartésienne», laquelle ne permet de caractériser de manière satisfaisante ni le pragmatisme (qui n'a guère le monopole de l'anticartésianisme), ni l'hégélianisme (qui ne se définit pas de manière essentielle par rapport au cartésianisme). Si la première approche pèche par une audace excessive, maniant sans complexe l'anachronisme et les raccourcis interprétatifs, la seconde pèche par une prudence apparente qui révèle, à plus ample examen, le caractère inadéquat du critère sur lequel repose son argumentation.

(suite)

démarche de Descartes pour démontrée de façon méthodologiquement conséquente; c'est un cheminement profond, intérieur, qui se manifeste naïvement» (ibid., p. 1391). Pour une présentation de la critique hégélienne de Descartes, voir mon ouvrage L'idéalisme hégélien (2013, ch. II, p. 125-130).

81 Voir Bernard Bourgeois, «Hegel et Descartes», dans Études hégéliennes (1992, p. 349) : «Les commencements sont décisifs et vides. Tel Descartes pour Hegel». 
De tels échecs n'excluent certes pas que d'autres stratégies plus convaincantes soient possibles ${ }^{82}$, mais ils invitent néanmoins à faire montre d'une certaine prudence à l'égard de la tentation de vouloir proposer une interprétation générale du rapport du pragmatisme à Hegel. Le nominalisme historique doit ici reprendre ses droits : face à cette «rage philosophique de la généralisation» ${ }^{83}$ qui s'empare volontiers des chantres du «pragmatisme hégélien», il semble plus opportun de substituer l'étude de cas singuliers, eux-mêmes dûment replacés dans leur contexte intellectuel et culturel respectif, à un diagnostic (prétendument) universel ${ }^{84}$. Fort heureusement, nous ne manquons pas de supports textuels pour cela : Peirce, James, Royce, Dewey, Mead, Rorty et Brandom ont eux-mêmes explicitement thématisé des perspectives aussi originales qu'instructives sur la manière de se confronter à Hegel en «pragmatiste» ${ }^{85}$. Il est peu probable que la comparaison de ces diverses confrontations singulières à l'auteur de la Phénoménologie de l'esprit révèle un «noyau» commun permettant de répondre, sous une forme unitaire et définitive, à la question initiale. Mais une telle diversité, loin d'être un défaut, est à la mesure de la richesse foisonnante d'un courant philosophique en incessante transformation, qui n'a jamais eu la vocation de se scléroser sous la forme d'une école partageant des dogmes figés ${ }^{86}$. D'un point de vue pragmatiste, la

82 Voir notamment la tentative faite par Terry Pinkard de reconstruire un «pragmatisme» hégélien à l'aune du problème de l'autorité normative de la raison dans «Le pragmatisme fut-il le successeur de l'idéalisme?» (trad. J.-M. Buée et L. Mérigonde, dans «Hegel pragmatiste?» [Buée et al., 2008]), ainsi que dans l'ensemble de ses ouvrages.

Nietzsche, Humain trop humain II, «Opinions et sentences mêlées», §5.

On peut à cet égard s'inspirer de la reconstruction historique proposée par Richard Bernstein dans son article «Hegel and Pragmatism», repris dans The Pragmatic Turn (2010). Cette approche contextualiste des relations entre Hegel et le pragmatisme est déjà particulièrement prégnante dans les travaux portant sur l'héritage hégélien de Dewey. Voir en particulier James Scott Johnston, John Dewey's Earlier Logical Theory (2014, ch. 2); John R. Shook et James A. Good, John Dewey's Philosophy of Spirit (2010); Emmanuel Renault, «Dewey's Relations to Hegel» (2016).

85 Au sujet de Rorty et Brandom, voir Olivier Tinland, «Le serpent et la tortue. Portraits post-sellarsiens de Hegel en "grand adversaire de 'l'immédiateté"”) (2012).

En outre, il serait inconséquent d'espérer dissiper l'équivocité d'un label philosophique aussi récent. Comme le font remarquer Robert B. Talisse et Scott F. Aitkin, «l'absence de définition du pragmatisme ne résulte pas d'un quelconque manque de précision de la part des philosophes pragmatistes; il est plutôt la conséquence $\mathrm{du}$ fait que le pragmatisme est comparativement un phénomène nouveau sur la scène philosophique. Cela fait seulement un peu plus de cent ans que le terme a été utilisé à l'écrit pour la première fois dans un contexte philosophique. Dans 
question «Hegel est-il pragmatiste?» est susceptible de recevoir autant de réponses qu'il y aura de «situations problématiques» requérant la «reconstruction» du questionnement pragmatiste, et par contrecoup la reconstruction du sens et de la pertinence de la pensée hégélienne pour le pragmatisme.

\section{Références bibliographiques}

Belaval, Yvon

1978 Leibniz critique de Descartes, Paris, Gallimard.

Bernstein, Richard

2010 The Pragmatic Turn, Malden, Polity Press.

Berthelot, René

1911 Un romantisme utilitaire. Étude sur le mouvement pragmatiste, Paris, Felix Alcan.

Bourgeois, Bernard

1992 Études hégéliennes, Paris, Presses universitaires de France.

Brandom, Robert

2002 Tales of the Mighty Dead, Cambridge (MA), Harvard University Press. Brandom, Robert

2011 Perspectives on Pragmatism, Cambridge (MA), Harvard University Press.

Brandom, Robert

2019 A Spirit of Trust. A Reading of Hegel's Phenomenology, Cambridge, Belknap Press.

Buée, Jean-Michel et al., dir.

2008 «Hegel pragmatiste?», Philosophie, nº 99.

(suite)

l'histoire de la philosophie, un siècle n'est guère suffisant pour permettre à une philosophie de se constituer en un programme intellectuel unifié. Par conséquent, il nous serait difficile d'énoncer avec précision la signification des termes utilisés pour désigner les philosophies qui sont apparues au $\mathrm{XX}^{\mathrm{e}}$ siècle : des termes tels qu'existentialisme, phénoménologie ou philosophie analytique comportent une ambiguïté analogue. Nous pouvons bien repérer des individus, des textes et des arguments qui caractérisent plus ou moins chacun de ces termes, mais nous ne pouvons en dire davantage. Il est possible qu'à un siècle ultérieur nous soyons capables de délimiter plus précisément la signification du pragmatisme, ainsi que nous le faisons pour identifier comme des programmes philosophiques spécifiques l'idéalisme absolu, l'empirisme britannique ou le rationalisme cartésien. Mais au point où nous en sommes, cette ambiguïté ne saurait être dissipée. Une telle situation n'a rien de déplorable. La résistance du pragmatisme à toute définition précise est un signe de sa vitalité, qui indique qu'il s'agit d'une philosophie vivante et non d'une relique historique» (Pragmatism. A Guide for the Perplexed [2008, p. 3]). 
Deledalle, Gérard

1967 L'idée d'expérience dans la philosophie de Dewey, Paris, Presses universitaires de France.

Gimmler, Antje

2004 «Pragmatic Aspects of Hegel's Thought», dans W. Egginton et M. Sandbothe, dir., The Pragmatist Turn in Philosophy, New York (NY), State University of New York Press, p. 47-66.

Gori, Pietro

2016 Il pragmatismo di Nietzsche. Saggi sul pensiero prospettivistico, Milano/Udine, Mimesis.

Granier, Jean

1966 Le problème de la vérité dans la philosophie de Nietzsche, Paris, Le Seuil.

Haack, Susan

1998 Manifesto of a Passionate Moderate. Unfashionable Essays, Chicago/ Londres, The University of Chicago Press.

Habermas, Jürgen

1973 La technique et la science comme “idéologie”, trad. J.-R. Ladmiral, Paris, Gallimard.

Habermas, Jürgen

2001 Vérité et justification, trad. R. Rochlitz, Paris, Gallimard.

Hegel, G.W.F.

1970 Encyclopédie des sciences philosophiques, t. I. La science de la logique [Enc. $1830-S L]$, trad. B. Bourgeois, Paris, Vrin.

Hegel, G.W.F.

1985 Leçons sur l'histoire de la philosophie, t. VI, trad. P. Garniron, Paris, Vrin.

Hegel, G.W.F.

1991 Notes et fragments. Iéna 1803-1806, trad. C. Colliot-Thélène et al., Paris, Aubier.

Hegel, G.W.F.

2004 Encyclopédie des sciences philosophiques, t. II. Philosophie de la nature, trad. B. Bourgeois, Paris, Vrin.

Hegel, G.W.F.

2006 Phénoménologie de l'esprit [Phéno.], trad. B. Bourgeois, Paris, Vrin.

Hegel, G.W.F.

2007 Leçons sur la logique. Berlin 1831, trad. J.-M. Buée et D. Wittmann, Paris, Vrin.

Hegel, G.W.F.

2013 Principes de la philosophie du droit, $3^{\mathrm{e}}$ édition, trad. J.-F. Kervégan, Paris, Presses universitaires de France.

Hegel, G.W.F.

2015 Science de la logique, livre premier, L'être [SL 1], trad. B. Bourgeois, Paris, Vrin. 
Hegel, G.W.F.

2016 Science de la logique, livre troisième, Le concept, trad. B. Bourgeois, Paris, Vrin.

Hildebrand, David

2003 Beyond Realism and Antirealism: John Dewey and the Neopragmatists, Nashville (TN), Vanderbilt University Press.

Honneth, Axel

2008 Les pathologies de la liberté. Une réactualisation de la philosophie du droit de Hegel, trad. F. Fischbach, Paris, La Découverte.

Horstmann, Rolf-Peter

1999 «What is Hegel's Legacy and What Should we Do With It?» European Journal of Philosophy, vol. $7, \mathrm{n}^{\mathrm{o}} 2$.

Houlgate, Stephen

2005 An Introduction to Hegel: Freedom, Truth and History, $2^{\mathrm{e}}$ édition, Oxford, Blackwell.

Houlgate, Stephen

2006 The Opening of Hegel's 'Logic', West Lafayette (IN), Purdue University Press.

Hume, David

2004 Essais et traités sur plusieurs sujets, trad. M. Malherbe, Paris, Vrin.

Johnston, James Scott

2014 John Dewey's Earlier Logical Theory, New York (NY), SUNY Press.

Kojève, Alexandre

1947 Introduction à la lecture de Hegel, Paris, Gallimard.

Labarrière, Pierre-Jean

1979 Introduction à une lecture de la Phénoménologie de l'esprit de Hegel, Paris, Aubier.

Lebrun, Gérard

1972 La patience du Concept. Essai sur le Discours hégélien, Paris, Gallimard.

Lovejoy, Arthur

1908 «The Thirteen Pragmatisms», The Journal of Philosophy, vol. V, n ${ }^{\circ} 1$. Malachowski, Alan, dir.

2013 The Cambridge Companion to Pragmatism, Cambridge, Cambridge University Press.

Merleau-Ponty, Maurice

1948 Sens et non-sens, Paris, Nagel.

Misak, Cheryl, dir.

2007 New Pragmatists, Oxford, Oxford University Press.

Mounce, H.O.

1997 The Two Pragmatisms: From Peirce to Rorty, Londres, Routledge.

Nuzzo, Angelica

2010 Hegel and the Analytic Tradition, Londres, Continuum. 
Okrent, Mark

1988 Heidegger's Pragmatism. Understanding, Being, and the Critique of Metaphysics, Ithaca (NY)/Londres, Cornell University Press.

Peirce, Charles Sanders

1931-1958 Collected Papers of C. S. Peirce, Cambridge (MA), Harvard University Press.

Peirce, Charles Sanders

2002 Pragmatisme et pragmaticisme. Euvres philosophiques, vol. 1, trad. Cl. Tiercelin et P. Thibaud, Paris, Le Cerf.

Perinetti, Dario et Marie-Andrée Ricard, dir.

2009 La Phénoménologie de l'esprit de Hegel : lectures contemporaines, Paris, Presses universitaires de France.

Redding, Paul

2007 Hegel and the Return of Hegelian Thought, New York (NY), Cambridge University Press.

Renault, Emmanuel

2016 «Dewey's Relations to Hegel», Contemporary Pragmatism, $\mathrm{n}^{\circ} 13$.

Rockmore, Tom

2005 Hegel, Idealism, and Analytic Philosophy, New Haven (CT)/Londres, Yale University Press.

Rorty, Richard

1993a Conséquences du pragmatisme, trad. J.-P. Cometti, Paris, Le Seuil.

Rorty, Richard

1993b Contingence, ironie et solidarité, trad. P.-E. Dauzat, Paris, Armand Colin.

Rorty, Richard

1994 Objectivisme, relativisme et vérité, trad. J.-P. Cometti, Paris, Presses universitaires de France.

Rorty, Richard

1995 Essais sur Heidegger et autres écrits, trad. J.-P. Cometti, Paris, Presses universitaires de France.

Rorty, Richard

1998 Truth and Progress, Cambridge, Cambridge University Press.

Rorty, Richard

2007 Philosophy as Cultural Politics, Cambridge, Cambridge University Press.

Rorty, Richard

2017 La philosophie et le miroir de la nature, trad. T. Marchaisse, Paris, Le Seuil.

Ryle, Gilbert

1949 The Concept of Mind, Londres, Hutchinson University Library.

Shook, John R. et James A. Good

2010 John Dewey's Philosophy of Spirit, New York (NY), Fordham University Press. 
Skorupski, John

2006 Why Read Mill Today? Londres/New York (NY), Routledge.

Soulez, Antonia, dir.

2010 Manifeste du Cercle de Vienne et autres écrits, $2^{\mathrm{e}}$ édition, Paris, Vrin.

Stern, Robert

2009 Hegelian Metaphysics, Oxford, Oxford University Press.

Talbot, Ellen Bliss

1907 «The Philosophy of Fichte in its Relation to Pragmatism», The Philosophical Review, vol. 16.

Talisse, Robert B. et Scott F. Aitkin

2008 Pragmatism. A Guide for the Perplexed, Londres, Continuum.

Taylor, Charles

1998 Hegel et la société moderne, trad. P. Desrosiers, Paris, Le Cerf.

Tinland, Olivier

2003 Hegel. Maîtrise et servitude, Paris, Ellipses.

Tinland, Olivier, dir.

2005 Lectures de Hegel, Paris, Livre de Poche.

Tinland, Olivier

2012 «Le serpent et la tortue. Portraits post-sellarsiens de Hegel en "grand adversaire de 'l'immédiateté"'》, Les Études philosophiques, 2012/4 $\left(n^{\circ} 103\right)$.

Tinland, Olivier

2013 L'idéalisme hégélien, Paris, CNRS-Éditions.

Wahl, Jean

1929 Le malheur de la conscience dans la philosophie de Hegel, Paris, Rieder. 